\title{
CTKG: A Knowledge Graph for Clinical Trials
}

\author{
Ziqi Chen ${ }^{1 \dagger}$, Bo Peng ${ }^{1 \dagger}$, Vassilis N. loannidis ${ }^{2}$, Mufei $\mathrm{Li}^{3}$, George Karypis ${ }^{2}$, and Xia Ning ${ }^{1 *}$
}

${ }^{1}$ The Ohio State University

${ }^{2}$ Amazon Web Services Al

${ }^{3}$ Amazon Web Services Shanghai Al Lab

*corresponding: ning.104@osu.edu

†These authors contributed equally to this work.

\begin{abstract}
Effective and successful clinical trials are essential in developing new drugs and advancing new treatments. However, clinical trials are very expensive and easy to fail. The high cost and low success rate of clinical trials motivate research on inferring knowledge from existing clinical trials in innovative ways for designing future clinical trials. In this manuscript, we present our efforts on constructing the first publicly available Clinical Trials Knowledge Graph, denoted as CTKG. CTKG includes nodes representing medical entities in clinical trials (e.g., studies, drugs and conditions), and edges representing the relations among these entities (e.g., drugs used in studies). Our embedding analysis demonstrates the potential utilities of CTKG in various applications such as drug repurposing and similarity search, among others.
\end{abstract}

\section{Introduction}

Clinical trials are studies aiming at determining the safety and efficacy of interventions, treatments or investigational drugs on human subjects ${ }^{1}$. Effective and successful clinical trials are essential in developing new drugs and advancing new treatments ${ }^{2}$. However, clinical trials are very expensive. As reported in Sertkaya et al. ${ }^{3}$, the average cost of a single phase in clinical trials ranges from 1.4 million up to 52.9 million US dollars. In addition, the success rate of the clinical trials is considerably low. As reported in Wong et al. ${ }^{4}$, for certain therapeutic groups like Oncology, the overall success rate of clinical trials could be as low as $3.4 \%$. The high cost and low success rate of clinical trials motivate deliberate analysis of existing clinical trials, inferring knowledge from them, utilizing existing clinical trials in innovative ways, and accordingly carefully designing future clinical trials. The Access to Aggregate Content of ClinicalTrials.gov (AACT) database ${ }^{5}$ represents an effort in enhancing the accessibility and analysis of the clinical trial data. However, as a relational database, AACT is not formatted for the purpose of inferring new knowledge from existing clinical trials ${ }^{6}$. A Knowledge Graph (KG), instead, is a graph representation in which information entities are represented as nodes, and their relations are coded as edges connecting the corresponding nodes. In contrast to relational databases, $\mathrm{KG}$ has been proven ${ }^{7-10}$ to be an effective representation for knowledge inference purposes. Constructing a KG over clinical trial data is vital for advancing the analysis and research of clinical trials. In this manuscript, we present our work on constructing a such KG, referred to as Clinical Trials Knowledge Graph, denoted as CTKG, and also release CTKG to the research community to facilitate advanced research using clinical trial data. CTKG includes nodes representing medical entities (e.g., studies, drugs and conditions), and edges representing relations among these entities (e.g., drugs used in studies). Different from the recently released knowledge base ${ }^{11}$ that focuses only on extracting medical entities from the eligibility criteria in clinical trials, CTKG includes more medical entities (e.g., adverse events and outcomes) and also the relations among these entities. The rich information in CTKG could enable more biomedical applications (e.g., adverse drug event prediction, outcome prediction) than the existing knowledge base in clinical trials. Figure 1 presents the schema of CTKG. The detailed descriptions of nodes and edges in CTKG will be presented in Section "Nodes in CTKG". To the best of our knowledge, CTKG is the first publicly available clinical trials knowledge graph in the scientific research community. The results of the embedding analysis over CTKG demonstrate its potential utilities in various applications such as drug repurposing and similarity search, among others.

\section{Results}

\section{Clinical Trials Data}

The clinical trials data in CTKG is collected from the Access to Aggregate Content of ClinicalTrials.gov (AACT) database. AACT is a publicly available relational database, which contains the information of every clinical trial registered in ClinicalTrials.gov, and is updated on a daily basis. In AACT, each clinical trial, also referred to as a study, is associated with a unique National Clinical Trial (NCT) ID, and all the information of a clinical trial is stored in 45 different tables. For example, information 
representing the medications, procedures and other actions provided or conducted in a clinical trial is stored in two tables: "interventions" and "browse interventions"; information representing the measurements used to evaluate the safety and efficacy of drugs or procedures studied in clinical trials is stored in the table "outcomes." All the tables and their schemas are available in https://aact.ctti-clinicaltrials.org/. Currently, CTKG includes 8,210 studies registered in the ClinicalTrials.gov between 1999 and 2020, and will be updated with new studies in the future. It includes only studies that have drug interventions and reported outcomes.

Note that CTKG does not include all the tables in AACT. For example, CTKG does not include tables such as "Sponsors", "Overall officials" and "Result contacts" because they are not directly related to the design and results of clinical trials, and including them may not significantly benefit the knowledge graph in analyzing the relations among medical entities. Other AACT tables such as "Provided documents" and "Documents" contain the links to detailed study protocols, informed consent forms and statistical analysis plans, etc. These documentations have rich textual information that might be complementary to the structural relations represented by CTKG. However, such information is highly specific to each individual clinical trial, and does not help establish new relations across clinical trials if no natural language processing is applied first, which by itself is highly non-trivial. Therefore, CTKG does not include such tables; instead, CTKG uses AACT's original study IDs so that all such information can still be retrieved from AACT if needed. CTKG does not include other AACT tables such as "Calculated values", "Design outcomes" and "Design group interventions" because information in such tables is already included in other tables that CTKG includes. Table 1 summarizes the AACT tables that are included and are not included in CTKG.

\section{CTKG Schema}

Figure 1 presents the schema of CTKG. The schema presents the different information entities involved in clinical trials, represented as nodes, and the relations among them, represented as edges. There are 18 types of nodes and 21 types of edges in CTKG. Each node and edge type has attributes describing the properties of the nodes and edges. The statistics of different nodes and edges are presented in Table 2 and Table 3, respectively. Detailed descriptions of node and edge attributes are available in the online documentation of CTKG: https://github.com/ninglab/CTKG/blob/main/Schema.pdf.

\section{Nodes in CTKG}

Each study node represents a clinical trial and is associated with the primary properties of that clinical trial as node attributes. The properties of each study node describe the purposes, phases and the protocols of the corresponding clinical trial. Each study node links to condition nodes, drug nodes, outcome nodes and multiple types of group nodes via one-to-many relationships. Each condition node describes a disease or syndrome that is extracted from the AACT and studied by some clinical trials.

Each drug-term node represents the drug used in clinical trials, and is identified by the extracted drug mention (Section "Drug Mentions and Normalization"). The drug-term nodes connect with study nodes via StudiedDrug and UsedDrug relations. The StudiedDrug relation connects studies and drug terms that are studied in at least one study group of the corresponding clinical trial, and the UsedDrug relation connects studies and the auxiliary drug terms such as pain reducers. Please refer to Section "Drug Mentions and Normalization" for more details.

Each outcome node represents an outcome measure used to evaluate the efficacy of interventions in the clinical trials, and has the name and the description of the outcome measure as attributes. Each outcome node is connected to a study node, representing that this specific outcome is used within the study. Note that unlike the condition node linking to multiple study nodes, each outcome node links to a unique study node. This is due to the complexity and the diversity of outcome measures, which makes it difficult to be shared across multiple study nodes. Each outcome node also links to one cluster-outcome node and multiple standard-outcome nodes. The connection between the outcome node and the cluster-outcome node represents that the name of the outcome can be assigned to the cluster represented by the cluster-outcome node, while the connection between the outcome node and the standard-outcome node represents that the name or the description of the outcome contains the standard outcome measure. Please refer to Section "Outcome Extraction and Outcome Clustering" for more details

Each group node represents a study arm or a comparison group, that is, a group of participants who receive a specific intervention. There are multiple types of group nodes as follows:

- event-group node. The information described by each event-group node is the number of participants within the group affected by specific types of adverse events. Each event-group node is connected to multiple drug nodes representing the drugs used in the event group, and adverse-event nodes representing the specific adverse events that occurred in the event group. Each adverse-event node also links to an organ-system node representing the affected organ system.

- baseline-group node. Each baseline-group node represents a group of participants with their demographic attributes (e.g., "Age" and "Ethnicity") or study-specific attributes (e.g., "Baseline Modified Gingival Index"). Each baseline-group node is connected to one or multiple baseline-record nodes. 
- drop-group node. Each drop-group node represents a group of participants with their withdrawal information. Each drop-group node is connected to one or multiple period nodes. Each period node represents an interval of the study (e.g., "First Intervention" and "Part 1: Treatment Period 1"), and has attributes describing the number of participants at the beginning and the end of the period. Each period node can link to multiple drop-record nodes. Each drop-record node includes a withdrawal reason and documents the number of the participants in the group withdrawing with this reason in a period.

- outcome-group node. An outcome-group node has the information on the efficacy of the studied interventions on the participants. The efficacy is evaluated by different outcome measures and analyzed by different statistical test methods with the measurements. Other nodes related to the efficacy measures of interventions are as below:

- method node. Each method node represents a method of statistical hypothesis testing that is used to test the superiority of an intervention as compared with a control in a clinical trial. Each method node is connected to multiple outcome-analysis nodes, representing that the method is used to conduct the analyses. Please refer to Section "Statistical Analysis Method Normalization" for more details about the normalization of method names.

- outcome-measurement node. Each outcome-measurement node represents the measurement of a specific outcome measure (i.e., outcome node) on the corresponding group of participants. Each outcome-measurement node links to one outcome node and one outcome-group node.

- outcome-analysis node. Each outcome-analysis node represents a statistical analysis on a specific outcome measure by comparing multiple outcome groups with a statistical test method. Each outcome-analysis node links to one outcome node, one method node and multiple outcome-group nodes.

Note that the different types of group nodes for a study could represent the same participant group with different information. According to AACT, using a single group to uniquely represent a participant group in the study is impossible due to the complicated designs of clinical trials. Therefore, we followed AACT and used different types of group nodes to represent different types of information of the clinical trials.

\section{Embedding Analysis}

We conducted an embedding analysis to evaluate the quality of CTKG and demonstrate its utilities using DGL-KE ${ }^{12}$ based $^{2}$ on the Deep Graph Library (DGL ${ }^{13}$ ). Specifically, we employed a widely used KG embedding method TransE ${ }^{14}$ to generate embeddings for nodes in CTKG, that is, each node is represented by a vector of dimension 200. Generating embeddings for nodes in CTKG benefits various downstream tasks. For example, we could establish similarities among nodes using their embeddings. The similarities enable fast retrieval of nodes corresponding to similar medical entities and could facilitate applications such as drug repurposing and similar study retrieval.

In the analysis, we evaluated if CTKG enables high quality embeddings on nodes, for example, in CTKG, if study nodes with similar embeddings correspond to clinical trials studying similar diseases or drugs. Particularly, we identified the top-10 most similar pairs of study, drug-term and standard-outcome nodes, respectively, using cosine similarities in their embeddings as presented in Tables 4, 5, 6, respectively. In each table, the first column presents the cosine similarity of each node pair, and the last column presents the commonality of the nodes in the pairs. As presented in Table 4, the identified studies with similar embeddings all investigated similar drugs or diseases. For example, the embeddings of study nodes NCT00795769 and NCT01789255 are very similar (cosine similarity 0.840), and both of the studies are on preventing the side effects of the stem cell transplantation. The study nodes NCT01431274 and NCT01431287 also have similar embeddings (cosine similarity 0.826), and both of the studies evaluate the safety and efficacy of the drug combination between Tiotropium and Olodaterol in patients with Chronic Obstructive Pulmonary Diseas (COPD). We also found a similar trend in Tables 5 and 6. As presented in Table 5, the identified similar drug-term nodes all have commonalities. For example, the embeddings of durg-term nodes ABT-267 and Macrocyclic Compounds are very similar (cosine similarity 0.997), and both of the two drugs could be used to treat Hepatitis C Virus (HCV) infection ${ }^{15,16}$. In addition, the two drugs are commonly studied together as in study NCT01458535, NCT01464827 and NCT01563536, among other. In Table 6, Aspartate Aminotransferase is very similar with Alanine Aminotransferase in embeddings (cosine similarity 0.986 ), and both of the outcomes measure the amount of two enzymes made by liver in the blood and can be tested to check the liver damage. These results demonstrate the utilities of CTKG in the application of similar medical entity (e.g., study, drug and outcome) retrieval, which could benefit the design of new clinical trials ${ }^{17}$.

CTKG could also enable other potential applications such as adverse drug event prediction and outcome prediction, etc. Specifically, for the adverse drug event prediction, we could employ knowledge reasoning methods ${ }^{18}$ over CTKG, and infer new adverse events of drugs using the existing or predicted paths from drug-term nodes to adverse-event nodes in CTKG. For the outcome prediction, we could employ link prediction methods ${ }^{9,14}$ to infer new edges between study nodes and outcome nodes 
based on the existing ones in CTKG. Overall, CTKG could facilitate new knowledge discovery and benefit the design of new clinical trials, and also improve the success rate of future clinical trials.

\section{Discussion}

In this manuscript, we presented and released a new knowledge graph CTKG for clinical trials. We also described our methods in generating CTKG. We demonstrated the potential utilities of CTKG in drug repurposing and similarity search, among others, via embedding analysis over CTKG. Currently, CTKG only includes studies that have both drug interventions and reported outcomes. However, incomplete studies (e.g., studies not started or without reported outcomes), and studies without drug interventions (e.g., studies for devices) could also contain valuable knowledge for the design of future clinical trials. Therefore, we will enrich CTKG with more studies in the future research. In addition, current CTKG does not contain all the important information for drug discovery and development. For example, CTKG does not have the interactions between drugs/molecules and proteins/diseases, nor the interactions among proteins. Missing such information may limit the utilities of CTKG for a much wider range of applications (e.g., to predict if a new molecule for a disease can survive from clinical trials). In the future research, we will align CTKG with other knowledge bases ${ }^{10,19,20}$ and integrate more and diverse information into CTKG to enable more applications using CTKG.

\section{Methods}

\section{Adverse Event Normalization}

In AACT, we could find the adverse events (AE), represented by AE terms, happened among the participants in the "reported events" table. Many AE terms listed in the table could be mapped to the Medical Dictionary for Regulatory Activities (MedDRA $\left({ }^{21}\right)$. MedDRA $(\mathrm{B}$ is the international medical terminology developed under the auspices of the International Council for Harmonisation of Technical Requirements for Pharmaceuticals for Human Use (ICH). More specifically, we found 28,677 unique AE terms in which 13,995 terms could be directly mapped to the MedDRA dictionary. In CTKG, such terms are also

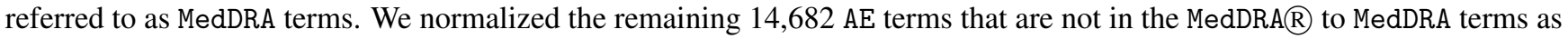
follows:

- We removed parenthesized contents (e.g., "Altered pitch perception (pitch seemed lower)"). The contents in parentheses are typically explanations or afterthoughts so removing them would not significantly affect the major meanings.

- We removed words or phrases that specify the auxiliary information (e.g., "left", "right", "Baseline Phase") or the time frame (e.g., "for 12 hours") of adverse events. We observed that these words or phrases are study-specific, and not in the MedDRA terms. For example, by removing the phrase "Baseline Phase", the AE term "Throat tightness - Baseline Phase" can be normalized to the MedDRA term "Throat tightness". The phrase "Baseline Phase" is given to specify the initial phase of assessment involving collection of initial data in the study, and thus unrelated to the adverse event itself.

- We removed the stop words and lemmatized AE terms using the NLTK library ${ }^{22}$, and Stanza NLP Library ${ }^{23}$, respectively.

- We mapped an AE term to its most similar MedDRA term if their edit distance is less than 4. For example, the adverse event term "Cholecyctitis" will be normalized to the MedDRA term "Cholecystitis". This process can correct simple misspellings.

After each step above, if the normalized AE term is a MedDRA term, we will stop the normalization. With the above normalization, we successfully normalized 7,296 AE terms to MedDRA terms. In total, we got 15,976 unique MedDRA terms and had 7,393 AE terms that cannot be normalized.

In order to construct a one-to-one mapping between the adverse events and the MedDRA terms, we used the MedDRA dictionary to further group multiple MedDRA terms of the same adverse event into a unique MedDRA term. According to the definition of MedDRA, each MedDRA term is assigned to one of the five hierarchical levels ${ }^{24}$. Specifically, the MedDRA terms with the lowest level (i.e., level 1), which are used to communicate the adverse events in practice, could correspond to the same adverse event. For example, "Eye itching" and "Ocular itching" are two MedDRA terms with level 1 and represent the same event. Such MedDRA terms corresponding to the same event have a common parent, which is a MedDRA term with level 2 (e.g., "itchy eyes" in the above example). Therefore, we normalized each MedDRA term with level 1 to its linked MedDRA term with level 2. In total, we converted 15,976 MedDRA terms into 11,153 more abstract MedDRA terms. Each term among these 11,153 MedDRA terms and 7,393 non-MedDRA terms represents an adverse event, which is further represented as an adverse-event node in CTKG. Note that due to the licensing restriction of MedDRA®, we didn't specify which adverse-event nodes represent MedDRA terms in CTKG and only kept the terms as the attribute of adverse-event nodes. 


\section{Drug Mentions and Normalization}

In AACT, the drugs used in studies (i.e., clinical trials) could be found in the intervention table, in which the "name" field stores the information about medicines and administrations used in each intervention. For example, we could find that the drug Naltrexone is used in the study NCT04322526 via its intervention "Naltrexone $50 \mathrm{Mg}$ Oral Tablet." In CTKG, we used Medical Text Indexer (MTI) ${ }^{25}$ to automatically extract drug mentions. MTI is developed by the National Library of Medicine (NLM) to recognize medical entities (e.g., anatomy, drugs and conditions) from plain text. We used this tool to extract drug mentions following 2 steps:

- We used MTI to automatically recognize all the medical entities from the interventions.

- We found drug entities from the medical entities recognized by MTI. Specifically, for each recognized entity, MTI will output its MeSH code if available. MeSH is a hierarchically-organized vocabulary from NLM to index and categorize biomedical and health-related information ${ }^{26}$. Given the MeSH code, we first identified entities with MeSH codes starting with character "D", which indicates drug entities (e.g., D02.241.223.701.430 for Ibuprofen). After that we removed the entities not representing specific drugs by excluding those with the MeSH code D26.310 (drug combination), D26 (pharmaceutical preparations), D23.101 (biomarkers) and D26.255 (dosage forms). We also noticed that a few recognized entities were not associated with MeSH codes. For these entities, we did a manual check and identified the ones representing specific drugs.

After the above 2 steps, there were still 1,775 unique interventions in which MTI did not find any drug mentions. For these interventions, we did a manual search and identified the drugs mentioned. Eventually, from the intervention table, we found 3,487 mentioned drugs in total. Among these drugs, 860 (24.7\%) of them are found manually. Most of the manually found drugs are investigational drugs (e.g., pf-06669571), or drugs mentioned in abbreviations (e.g., tvr and umec).

Besides the drugs in interventions, there were also drugs mentioned in the titles or descriptions of the study groups (e.g., event group). For example, from the title "tramadol/diclofenac 25/25", we could find the drugs Tramadol and Diclofenac. We also extracted drugs mentioned in the titles or descriptions of study groups to generate a complete list of drug mentions. Specifically, we first used the above 2 steps to automatically extract the mentioned drugs in titles and descriptions of study groups. For groups that we did not find any drugs automatically, we manually searched their titles and descriptions, and identified the mentioned drugs. In the end, we found 4,585 drug mentions from the interventions and the study groups.

From the drug mentions, we observed that one drug could be represented by different names. For example, the drug "losartan potassium" could be represented by its brand name "cozaar" or its generic name "losartan." Therefore, we normalized the drug mentions found in texts to normalized terms. Specifically, we first used MTI to map all the 4,585 drugs to their MeSH terms. For example, MTI could automatically map the drugs "losartan potassium", "cozaar" and "losartan" to the MeSH term "losartan." For the drugs that MTI can find their MeSH terms, the MeSH terms were used as their normalized terms. For the other drugs, if they are in abbreviations (e.g., tvr), we first found their full names (e.g., Telaprevir), and used the MeSH terms of their full names for normalization; if they are not in abbreviations, we used their generic names for normalization. We noticed that investigational drugs may not have generic names. For these drugs, their identifiers mentioned in studies (e.g., pf-06669571) were used as their normalized terms. After the normalization, the 4,585 drug mentions were normalized to 2,548 normalized terms. Each of the normalized term is represented as a drug-term node in CTKG.

\section{Statistical Analysis Method Normalization}

We observed that one statistical analysis method could be represented by different names in the table. For example, the method "paired $t$-test" could be represented as "paired $t$ test", "paired $t$-tests" and "paited $t$-test" in the table. Therefore, we normalized the names of the methods using the 3 steps as follows:

- We preprocessed the method names from the table by removing the space and punctuation in the text.

- We calculated the edit distance among the preprocessed names, and normalized the preprocessed names with edit distance less than 4 to a same normalized term. We also did a manual check to correct possible mis-normalization. For the names that will be normalized to a same term, we used the names with the highest frequency as the normalized term.

- We further refined the normalized terms by merging the terms with the same words. We noticed that after the second step, there were still normalized terms that represent the same method with the same words but of different orders. For example, the normalized terms "pairedttest" and "ttestpaired" represent the same method "paired $t$-test" with the same words but of different orders. We manually merged such terms to the one with the highest frequency.

After all the steps, we normalized the 1,299 unique method names mentioned in the table to 907 normalized terms. Each of the normalized terms is represented as a method node in CTKG. 


\section{Outcome Extraction and Outcome Clustering}

In AACT, the outcome measures used to test the effectiveness of the interventions could be found in the "title" or the "description" fields of the outcome table. Most of the titles in the outcome table are long phrases and could involve multiple standard outcome measures (e.g., in the title "Change From Baseline in Platelet Count and White Blood Cell Count", where "Platelet Count" and "White Blood Cell Count" represent standard outcome measures). The complex relations between the outcome titles and the standard outcome measures make it difficult to directly represent the outcomes with the extracted standard outcome measures. Therefore, we incorporated the identified standard outcome measures as nodes into CTKG and built connections between the outcome nodes and the involved standard-outcome nodes. Through such connections, we can infer which standard outcome measures are used in each study to assess the efficacy of interventions. We observed that some popular phrases within the titles or the descriptions of outcome records represent standardized assessment tools used to measure the outcome of clinical trials, for example, "Visual Analogue Scale" is a tool widely used as a measure for pain. Incorporating such standard outcome measures into the CTKG could enable the comparison on the outcome measurements across different studies, and also could provide a reference regarding the choice of standardized assessment tools in the design of clinical trials. Therefore, we extracted the phrases that could represent standard outcome measures as below:

- We found the abbreviations and identified the definitions of abbreviations from the titles or the descriptions of the outcomes using the Schwartz-Hearst algorithm ${ }^{27}$. We observed in the titles that many standard outcome measures are associated with their corresponding abbreviations. For example, we could identify the abbreviation "BI" and the corresponding definitions "Bleeding Index" from the outcome name "Gingival Health Measured by Bleeding Index (BI)."

- We kept only the definitions containing the following words: scale, index, score, test, questionnaire, value, count, inventory, assessment, level, rate. We observed that most standard outcome measures would contain such words (e.g., "Visual Analogue Scale", "Social Responsiveness Scale").

- We manually normalized different variants of the same standard outcome measures and removed the extracted phrases that are not outcome measures. We also manually added some popular standard outcome measures (e.g., "Overall Survival", "blood pressure", "triglyceride") that do not contain the above words or do not have any abbreviations.

All the extracted phrases are represented as the standard-outcome nodes in the CTKG. In the end, we got 492 standard-outcome nodes from 50,342 outcome records (i.e., $56.96 \%$ over all the 88,386 outcome records), and connected the standard-outcome nodes with the corresponding outcome nodes.

With the extracted standard outcome measures, there were still more than $40 \%$ of the outcome nodes not connected to any standard-outcome nodes. Therefore, to aggregate similar outcome nodes, we also grouped all the outcome titles (including those containing the standard outcome measures) into several clusters. Specifically, we represented each outcome title using its term frequency-inverse document frequency (TF-IDF) vectors. We then grouped the TF-IDF vectors of outcome titles using the CLUTO $^{28}$, a clustering toolkit, into 200 clusters. Each cluster is presented as a cluster-outcome node and has attributes describing the cluster size, that is, the number of outcomes within the cluster, and the most representative words of these outcomes. Specifically, for each cluster, the representative words of outcomes include 5 descriptive words and 5 discriminating words derived by CLUTO that can best describe or discriminate each cluster. Each word is associated with a percentage computed by CLUTO (details in its manual) which indicates the importance of this word with respect to describing or discriminating the cluster. We converted the descriptive words and the discriminating words as two attributes of each cluster, by combining the words and their corresponding percentages (e.g., "circumference 56.4\%, waist $43.0 \%$, head $0.1 \%$, abdominal $0.1 \%$,change $0.1 \% ")$.

\section{Data Availability}

The dataset generated and analyzed during the current study is released in GitHub: https://git hub. com/ninglab/CTKG.

\section{References}

1. Elkin, M. E. \& Zhu, X. Predictive modeling of clinical trial terminations using feature engineering and embedding learning. Sci. Rep. 11, 1-12 (2021).

2. Friedman, L. M., Furberg, C. D., DeMets, D. L., Reboussin, D. M. \& Granger, C. B. Fundamentals of clinical trials (Springer, 2015).

3. Sertkaya, A., Wong, H.-H., Jessup, A. \& Beleche, T. Key cost drivers of pharmaceutical clinical trials in the united states. Clin. Trials J. 13, 117-126 (2016). 
medRxiv preprint doi: https://doi.org/10.1101/2021.11.04.21265952; this version posted November 9, 2021. The copyright holder for this preprint (which was not certified by peer review) is the author/funder, who has granted medRxiv a license to display the preprint in perpetuity.

It is made available under a CC-BY 4.0 International license .

4. Wong, C. H., Siah, K. W. \& Lo, A. W. Estimation of clinical trial success rates and related parameters. Biostatistics 20, 273-286 (2019).

5. Tasneem, A. et al. The database for aggregate analysis of ClinicalTrials.gov (AACT) and subsequent regrouping by clinical specialty. PLOS ONE 7, e33677 (2012).

6. Macak, M., Stovcik, M. \& Buhnova, B. The suitability of graph databases for big data analysis: A benchmark. In International Conference on Internet of Things, Big Data and Security (IoTBDS) (2020).

7. Pujara, J., Miao, H., Getoor, L. \& Cohen, W. Knowledge graph identification. In International Semantic Web Conference (ISWC), 542-557 (Springer, 2013).

8. Ma, Y., Crook, P. A., Sarikaya, R. \& Fosler-Lussier, E. Knowledge graph inference for spoken dialog systems. In 2015 IEEE International Conference on Acoustics, Speech and Signal Processing (ICASSP), 5346-5350 (IEEE, 2015).

9. Ji, S., Pan, S., Cambria, E., Marttinen, P. \& Philip, S. Y. A survey on knowledge graphs: Representation, acquisition, and applications. IEEE Trans. Neural Netw. Learn. Syst. (2021).

10. Ioannidis, V. N. et al. DRKG - drug repurposing knowledge graph for covid-19. https://github.com/gnn4dr/DRKG/ (2020).

11. Liu, H., Chi, Y., Butler, A., Sun, Y. \& Weng, C. A knowledge base of clinical trial eligibility criteria. J. Biomed. Inform. 117, 103771 (2021).

12. Zheng, D. et al. DGL-KE: Training knowledge graph embeddings at scale. In Proceedings of the 43rd International ACM SIGIR Conference on Research and Development in Information Retrieval, SIGIR '20, 739-748 (Association for Computing Machinery, New York, NY, USA, 2020).

13. Wang, M. et al. Deep graph library: A graph-centric, highly-performant package for graph neural networks. arXiv preprint arXiv:1909.01315 (2019).

14. Bordes, A., Usunier, N., Garcia-Durán, A., Weston, J. \& Yakhnenko, O. Translating embeddings for modeling multirelational data. In Proceedings of the 26th International Conference on Neural Information Processing Systems - Volume 2, 2787-2795 (Curran Associates Inc., Red Hook, NY, USA, 2013).

15. Feld, J. J. et al. Treatment of HCV with ABT-450/r-ombitasvir and dasabuvir with ribavirin. N. Engl. J. Med. 370, 1594-1603 (2014).

16. Pillaiyar, T., Namasivayam, V. \& Manickam, M. Macrocyclic hepatitis C virus NS3/4A protease inhibitors: an overview of medicinal chemistry. Curr. Med. Chem. 23, 3404-3447 (2016).

17. Hao, T., Rusanov, A., Boland, M. R. \& Weng, C. Clustering clinical trials with similar eligibility criteria features. $J$. Biomed. Inform. 52, 112-120 (2014).

18. Chen, X., Jia, S. \& Xiang, Y. A review: Knowledge reasoning over knowledge graph. Expert. Syst. Appl. 141, 112948 (2020).

19. Santos, A. et al. Clinical knowledge graph integrates proteomics data into clinical decision-making. bioRxiv (2020).

20. Xu, J. et al. Building a PubMed knowledge graph. Sci. Data 7 (2020).

21. Brown, E. Medical dictionary for regulatory activities (MedDRA@). In Pharmacovigilance, 168-183 (John Wiley \& Sons, Ltd, 1999).

22. Bird, S., Klein, E. \& Loper, E. Natural Language Processing with Python (O’Reilly UK Ltd., 2009).

23. Zhang, Y., Zhang, Y., Qi, P., Manning, C. D. \& Langlotz, C. P. Biomedical and clinical english model packages for the stanza python NLP library. J. Am. Med. Inform. Assoc. 28, 1892-1899 (2021).

24. Yokotsuka, M., Aoyama, M. \& Kubota, K. The use of a medical dictionary for regulatory activities terminology (MedDRA) in prescription-event monitoring in japan (J-PEM). Int. J. Med. Inform. 57, 139-153 (2000).

25. Mork, J. G., Jimeno-Yepes, A., Aronson, A. R. et al. The NLM medical text indexer system for indexing biomedical literature.BioASQ@CLEF 1 (2013).

26. Chapman, D. Advanced search features of PubMed. J. Can. Acad. Child Adolesc. Psychiatry 18, 58 (2009).

27. Schwartz, A. S. \& Hearst, M. A. A simple algorithm for identifying abbreviation definitions in biomedical text. In Pac. Symp. Biocomput., 451-462 (World Scientific, 2003).

28. Karypis, G. Cluto: A clustering toolkit. Tech. Rep. 02-017, Dept. of Computer Science, University of Minnesota. (2002).

29. Anestopoulos, I. et al. Surface active agents and their health-promoting properties: Molecules of multifunctional significance. Pharmaceutics 12, 688 (2020). 
30. Lancaster, D., Lennard, L., Rowland, K., Vora, A. \& Lilleyman, J. Thioguanine versus mercaptopurine for therapy of childhood lymphoblastic leukaemia: A comparison of haematological toxicity and drug metabolite concentrations. Br. J. Haematol. 102, 439-443 (1998).

31. Clissold, S. P., Todd, P. A. \& Campoli-Richards, D. M. Imipenem/cilastatin. Drugs 33, 183-241 (1987).

32. Sumiyoshi, T., Jayathilake, K. \& Meltzer, H. Y. The effect of melperone, an atypical antipsychotic drug, on cognitive function in schizophrenia. Schizophr, Res. 59, 7-16 (2003).

33. Zhang, Y., Liu, J., Chen, X. Q. \& Chen, C. Y. O. Ubiquinol is superior to ubiquinone to enhance Coenzyme Q10 status in older men. Food Funct. 9, 5653-5659 (2018).

34. Wilck, M. B., Xu, Z. J., Stek, J. E. \& Lee, A. W. Safety and immunogenicity of a fully-liquid DTaP-IPV-Hib-HepB vaccine (Vaxelis) in premature infants. Hum. Vaccines Immunother. 17, 191-196 (2021).

35. Croxtall, J. D. \& Keating, G. M. Pneumococcal polysaccharide protein D-conjugate vaccine (Synflorix; PHiD-CV). Pediatr. Drugs 11, 349-357 (2009).

36. Bellandi, F. et al. Long-term efficacy and safety of propafenone and sotalol for the maintenance of sinus rhythm after conversion of recurrent symptomatic atrial fibrillation. Am. J. Cardiol. 88, 640-645 (2001).

37. Keller, J. E. \& Neale, E. A. The role of the synaptic protein snap- 25 in the potency of botulinum neurotoxin type A. J. Biol. Chem. 276, 13476-13482 (2001).

38. Williams, A. L. \& Hoofnagle, J. H. Ratio of serum aspartate to alanine aminotransferase in chronic hepatitis relationship to cirrhosis. Gastroenterology 95, 734-739 (1988).

39. Smolen, J. S. et al. Validity and reliability of the twenty-eight-joint count for the assessment of rheumatoid arthritis activity. Arthritis Rheum. 38, 38-43 (1995).

40. National Heart, Lung, and Blood Institute. Bethesda (MD): U.S. Department of Health and Human Services; Types of Blood Tests. https://www.nhlbi.nih.gov/health-topics/blood-tests\#Types. Accessed: 2021-10-24.

41. Therasse, P. et al. New guidelines to evaluate the response to treatment in solid tumors. J. Natl. Cancer Inst. 92, 205-216 (2000).

42. Baum, N., Dichoso, C. C. \& Carlton, C. Blood urea nitrogen and serum creatinine. Urology 5, 583-588 (1975).

43. Radin, M. S. Pitfalls in hemoglobin a1c measurement: When results may be misleading. J. Gen. Intern. Med. 29, 388-394 (2013).

44. Inoue, E., Yamanaka, H., Hara, M., Tomatsu, T. \& Kamatani, N. Comparison of disease activity score (DAS)28- erythrocyte sedimentation rate and DAS28- C-reactive protein threshold values. Annals Rheum. Dis. 66, 407-409 (2007).

\section{Acknowledgement}

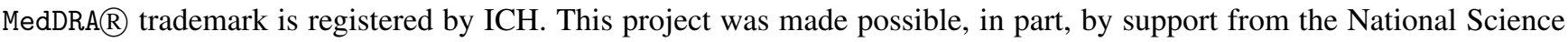
Foundation grant numbers (IIS-1855501, X.N.; IIS-1827472, X.N.; IIS-2133650, X.N.), an AWS Machine Learning Research Award (X.N.). Any opinions, findings, and conclusions or recommendations expressed in this material are those of the authors, and do not necessarily reflect the views of the funding agencies.

\section{Author contributions statement}

X.N. and G.K. conceived the research. All the authors designed the research; Z.C. and B.P. conducted the research, including data curation, formal analysis, methodology design and implementation, result analysis and visualization; X.N. supervised and mentored Z.C. and B.P.; Z.C. and B.P. drafted the original manuscript; X.N. edited and revised the manuscript; V.I.L., M.L. and G.K. provided comments; all authors reviewed the final manuscript.

\section{Competing Interests Statement}

G.K. and V.I.L. are employed by the company Amazon Web Services AI. M.L. is employed by the company Amazon Web Services Shanghai AI Lab. The remaining authors declare that the research was conducted in the absence of any commercial or financial relationships that could be construed as a potential conflict of interest. 


\section{Figure}

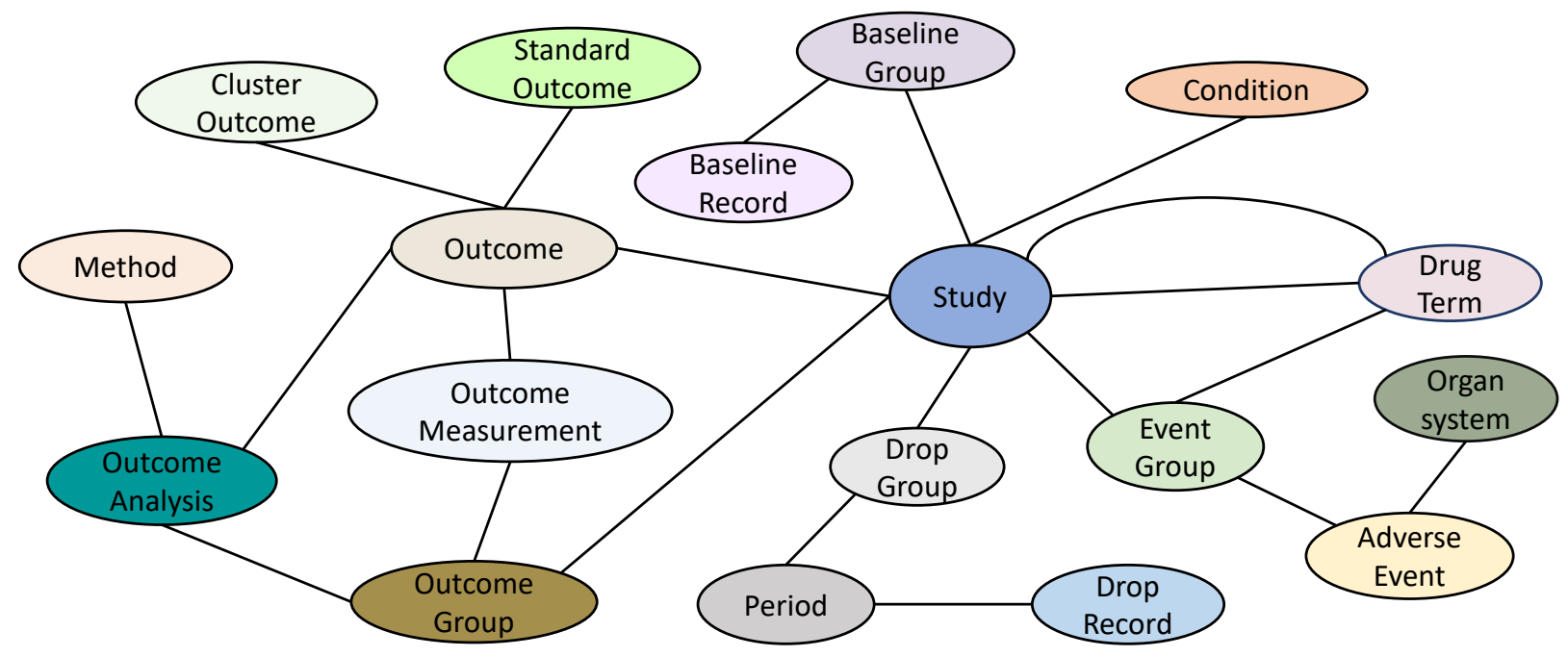

Figure 1. Schema of CTKG

\section{Tables}

Table 1. AACT tables included and not included in CTKG

\begin{tabular}{ll}
\hline Included in CTKG & Not included in CTKG \\
\hline Baseline counts & Brief summaries \\
Baseline measurements & Calculated values \\
Browse conditions & Central contacts \\
Browse intervention & Countries \\
Conditions & Design group interventions \\
Designs & Design groups \\
Drop withdrawals & Design outcomes \\
Eligibilities & Detailed descriptions \\
Interventions & Documents \\
Id information & Facilities \\
Milestones & Facilities contacts \\
Outcome measure & Facility investigator \\
Outcome analyses & Intervention other names \\
Outcome analysis groups & Ipd information types \\
Outcomes & Keywords \\
Participant flows & Links \\
Reported events & Overall officials \\
Result groups & Pending results \\
Studies & Provided documents \\
Study references & Responsible parties \\
& Result agreements \\
& Result contacts \\
& Sponsors \\
\hline
\end{tabular}


Table 2. Statistics of node types in CTKG

\begin{tabular}{lcr}
\hline Node type & Is study specific? & Statistics \\
\hline Study & Yes & 8,210 \\
Condition & No & 1,394 \\
Drug Term & No & 2,548 \\
\hline Event Group & Yes & 22,725 \\
Adverse Event & No & 18,546 \\
Organ & No & 27 \\
\hline Baseline Group & Yes & 27,068 \\
Baseline Record & Yes & 315,533 \\
\hline Drop Group & Yes & 22,272 \\
Period & Yes & 34,330 \\
Drop Record & Yes & 123,627 \\
\hline Outcome Group & Yes & 32,499 \\
Method & No & 907 \\
Outcome Measurement & Yes & 690,626 \\
Outcome Analysis & Yes & 107,294 \\
Outcome & Yes & 88,386 \\
Standard Outcome & No & 492 \\
Cluster Outcome & No & 200 \\
\hline
\end{tabular}

Table 3. Statistics of relation types in CTKG

\begin{tabular}{|c|c|c|c|c|c|}
\hline Relation type & Node type 1 & \#Node 1 & Node type 2 & \#Node 2 & \#Relations \\
\hline Study-Condition & Study & 8,210 & Condition & 1,394 & 17,259 \\
\hline Study-EventGroup & Study & 8,172 & Event Group & 22,725 & 22,725 \\
\hline Study-BaselineGroup & Study & 8,209 & Baseline Group & 27,068 & 27,068 \\
\hline Study-DropGroup & Study & 8,210 & Drop Group & 22,272 & 22,272 \\
\hline Study-OutcomeGroup & Study & 8,210 & Outcome Group & 32,499 & 32,499 \\
\hline Study-Outcome & Study & 8,210 & Outcome & 88,386 & 88,386 \\
\hline Study-StudiedDrug & Study & 8,169 & Drug Term & 2,373 & 23,188 \\
\hline Study-UsedDrug & Study & 3,090 & Drug Term & 1,105 & 6,113 \\
\hline Drug-EventGroup & Drug Term & 2,201 & Event Group & 21,790 & 33,453 \\
\hline EventGroup-AdverseEvent & Event Group & 20,571 & Adverse Event & 18,546 & 966,450 \\
\hline AdverseEvent-Organ & Adverse Event & 18,546 & Organ & 27 & 18,546 \\
\hline BaselineGroup-BaselineRecord & Baseline Group & 27,068 & Baseline Record & 315,533 & 315,533 \\
\hline DropGroup-Period & Drop Group & 22,272 & Period & 34,330 & 34,330 \\
\hline Period-DropRecord & Period & 25,956 & Drop Record & 123,627 & 123,627 \\
\hline OutcomeGroup-OutcomeMeasurement & Outcome Group & 32,240 & Outcome Measurement & 690,541 & 690,541 \\
\hline OutcomeGroup-OutcomeAnalysis & Outcome Group & 23,923 & Outcome Analysis & 107,294 & 209,314 \\
\hline OutcomeAnalysis-Method & Outcome Analysis & 91,463 & Method & 907 & 91,463 \\
\hline Outcome-OutcomeAnalysis & Outcome & 45,689 & Outcome Analysis & 107,294 & 107,294 \\
\hline Outcome-OutcomeMeasurement & Outcome & 85,905 & Outcome Measurement & 690,626 & 690,626 \\
\hline Outcome-ClusterOutcome & Outcome & 88,244 & Cluster Outcome & 200 & 88,244 \\
\hline Outcome-StandardOutcome & Outcome & 50,342 & Standard Outcome & 492 & 58,819 \\
\hline
\end{tabular}

Columns represent: "Relation type": the type of relation; "Node type 1": the type of head nodes in the relations; "\#Node 1 ": the number of unique head nodes with the relations; "Node type 2": the type of tail nodes in the relations; "\#Node 2": the number of unique tail nodes with the relations; "\#Relations": the number of relations of a relation type. 
Table 4. Top-10 Similar Study Nodes

\begin{tabular}{cll}
\hline Cosine Similarity & Similar Study Nodes & Commonality \\
\hline 0.840 & $\begin{array}{l}\text { NCT00795769 } \\
\text { NCT01789255 }\end{array}$ & $\begin{array}{l}\text { Both of the studies are on preventing side effects following the stem cell } \\
\text { transplantation. }\end{array}$ \\
\hline 0.826 & $\begin{array}{l}\text { NCT01431274 } \\
\text { NCT01431287 }\end{array}$ & $\begin{array}{l}\text { Both of the studies evaluate the efficacy of Tiotropium and Olodaterol in } \\
\text { patients with Chronic Obstructive Pulmonary Disease (COPD). }\end{array}$ \\
\hline 0.825 & $\begin{array}{l}\text { NCT00137111 } \\
\text { NCT00866307 }\end{array}$ & $\begin{array}{l}\text { Both of the studies investigate therapies for the acute lymphoblastic } \\
\text { leukemia. }\end{array}$ \\
\hline 0.807 & $\begin{array}{l}\text { NCT00782509 } \\
\text { NCT00796653 }\end{array}$ & $\begin{array}{l}\text { Both of the studies evaluate the safety and efficacy of orally inhaled BI 1744 } \\
\text { CL in patients with COPD. }\end{array}$ \\
\hline 0.801 & $\begin{array}{l}\text { NCT02105688 } \\
\text { NCT02252016 }\end{array}$ & $\begin{array}{l}\text { Both of the studies evaluate the combination of MK-5172 and MK-8742 in } \\
\text { subjects with chronic Hepatitis C Viru (HCV) infection. }\end{array}$ \\
\hline 0.799 & $\begin{array}{l}\text { NCT00720109 } \\
\text { NCT00866307 }\end{array}$ & $\begin{array}{l}\text { Both of the studies investigate therapies for the acute lymphoblastic } \\
\text { leukemia. }\end{array}$ \\
\hline NCT01753866 & $\begin{array}{l}\text { Both of the studies evaluate AMG 145 on Low-Density Lipoprotein } \\
\text { Cholesterol (LDL-C) in combination with statin therapies. }\end{array}$ \\
\hline 0.786 & $\begin{array}{l}\text { NCT01964352 } \\
\text { NCT02006732 }\end{array}$ & $\begin{array}{l}\text { Both of the studies evaluate Tiotropium and Olodaterol fixed dose } \\
\text { combination in patients with moderate to severe COPD. }\end{array}$ \\
\hline \multirow{2}{*}{0.783} & $\begin{array}{l}\text { NCT00782210 } \\
\text { NCT00793624 }\end{array}$ & $\begin{array}{l}\text { Both of the studies evaluate the safety and efficacy of orally inhaled BI 1744 } \\
\text { CL in patients with COPD. }\end{array}$ \\
\hline NCT03478683 & $\begin{array}{l}\text { Both of the studies compare therapy FF/UMEC/VI with therapy } \\
\text { Budesonide/Formoterol plus Tiotropium in participants with COPD. }\end{array}$ \\
\hline
\end{tabular}

Table 5. Top-10 Similar Drug-Term Nodes

\begin{tabular}{|c|c|c|}
\hline Cosine Similarity & Similar Drug-Term Nodes & Commonality \\
\hline 0.997 & $\begin{array}{l}\text { ABT-267 } \\
\text { Macrocyclic Compounds }\end{array}$ & $\begin{array}{l}\text { Both ABT-267 and Macrocyclic Compounds could be used to } \\
\text { treat Hepatitis C Virus (HCV) infection }{ }^{15,16} \text {. }\end{array}$ \\
\hline 0.996 & $\begin{array}{l}\text { Pulmonary Surfactants } \\
\text { Surface-Active Agents }\end{array}$ & Pulmonary Surfactants is a type of Surface-Active Agents ${ }^{29}$. \\
\hline 0.995 & $\begin{array}{l}\text { Phenylethyl Alcohol } \\
\text { LY2216684 }\end{array}$ & $\begin{array}{l}\text { Phenylethyl Alcohol and LY2216684 are studied together in } \\
\text { study NCT00922636, NCT01243957 and NCT01380691. }\end{array}$ \\
\hline 0.994 & $\begin{array}{l}\text { Thioguanine } \\
\text { Mercaptopurine }\end{array}$ & $\begin{array}{l}\text { Thioguanine is a substitute of Mercaptopurine in treating } \\
\text { childhood lymphoblastic leukaemia }{ }^{30} \text {. }\end{array}$ \\
\hline 0.993 & $\begin{array}{l}\text { Cilastatin } \\
\text { Imipenem }\end{array}$ & $\begin{array}{l}\text { Cilastatin and Imipenem are commonly used together as a } \\
\text { treatment for serious infections }{ }^{31} \text {. }\end{array}$ \\
\hline 0.985 & $\begin{array}{l}\text { Metylperon } \\
\text { Butyrophenones }\end{array}$ & $\begin{array}{l}\text { Metylperon is an atypical antipsychotic of the Butyrophenone } \\
\text { chemical class }^{32} \text {. }\end{array}$ \\
\hline 0.983 & $\begin{array}{l}\text { Ubiquinone } \\
\text { Coenzyme Q10 }\end{array}$ & Ubiquinone is a form of Coenzyme Q10 ${ }^{33}$. \\
\hline 0.982 & $\begin{array}{l}\text { PHiD-CV Vaccine } \\
\text { VAXELIS }\end{array}$ & Both of the drug terms are vaccines for diphtheria ${ }^{34,35}$. \\
\hline 0.982 & $\begin{array}{l}\text { Propafenone } \\
\text { Sotalol }\end{array}$ & $\begin{array}{l}\text { Both Propafenone and Sotalol could maintain sinus rhythm for } \\
\text { patients with recurrent symptomatic atrial fibrillation }{ }^{36} \text {. }\end{array}$ \\
\hline 0.980 & $\begin{array}{l}\text { SNAP25 Protein } \\
\text { Acetylcholine }\end{array}$ & $\begin{array}{l}\text { SNAP25 Protein could block Acetylcholine releasing at the } \\
\text { neuromuscular junction }{ }^{37} \text {. }\end{array}$ \\
\hline
\end{tabular}


Table 6. Top-10 Similar Standard-Outcome Nodes

\begin{tabular}{|c|c|c|}
\hline Cosine Similarity & Similar Standard-Outcome Nodes & Commonality \\
\hline 0.986 & $\begin{array}{l}\text { Aspartate Aminotransferase } \\
\text { Alanine Aminotransferase }\end{array}$ & $\begin{array}{l}\text { Both of the standard outcomes are enzymes that are tested to } \\
\text { check the liver damage }{ }^{38} \text {. }\end{array}$ \\
\hline 0.955 & $\begin{array}{l}\text { Swollen Joint Count } \\
\text { Tender Joint Count }\end{array}$ & $\begin{array}{l}\text { Both of the standard outcomes are used to assess patients with } \\
\text { rheumatoid arthritis }{ }^{39} \text {. }\end{array}$ \\
\hline 0.952 & $\begin{array}{l}\text { Calcium } \\
\text { Potassium }\end{array}$ & $\begin{array}{l}\text { Both of the standard outcomes are electrolyte that can be tested } \\
\text { to monitor a range of medical conditions }{ }^{40} \text {. }\end{array}$ \\
\hline 0.952 & $\begin{array}{l}\text { Incomplete Response } \\
\text { Partial Response }\end{array}$ & $\begin{array}{l}\text { Both of the standard outcomes are used to assess the response } \\
\text { to treatment }{ }^{41} \text {. }\end{array}$ \\
\hline 0.946 & $\begin{array}{l}\text { Aspartate Aminotransferase } \\
\text { Blood Urea Nitrogen }\end{array}$ & $\begin{array}{l}\text { Both of the standard outcomes can be tested to check liver } \\
\text { damage }^{38,42} \text {. }\end{array}$ \\
\hline 0.941 & $\begin{array}{l}\text { Potassium } \\
\text { Blood Urea Nitrogen }\end{array}$ & $\begin{array}{l}\text { Both of the standard outcomes are included in basic metabolic } \\
\text { panel blood test }{ }^{40} \text {. }\end{array}$ \\
\hline 0.940 & $\begin{array}{l}\text { Calcium } \\
\text { Blood Urea Nitrogen }\end{array}$ & $\begin{array}{l}\text { Both of the standard outcomes are included in basic metabolic } \\
\text { panel blood test }{ }^{40} \text {. }\end{array}$ \\
\hline 0.930 & $\begin{array}{l}\text { Alanine Aminotransferase } \\
\text { Blood Urea Nitrogen }\end{array}$ & $\begin{array}{l}\text { Both of the standard outcomes can be tested to check kidney } \\
\text { damage }^{38,42} \text {. }\end{array}$ \\
\hline 0.930 & $\begin{array}{l}\text { Hemoglobin A1c } \\
\text { Hemoglobin }\end{array}$ & $\begin{array}{l}\text { Hemoglobin A1c represents the hemoglobin in the blood that } \\
\text { has glucose attached to it }{ }^{43} \text {. }\end{array}$ \\
\hline 0.923 & $\begin{array}{l}\text { Erythrocyte Sedimentation Rate } \\
\text { Disease Activity Score } 28\end{array}$ & $\begin{array}{l}\text { Disease Activity Score } 28 \text { can be calculated based on } \\
\text { Erythrocyte Sedimentation Rate }{ }^{44} \text {. }\end{array}$ \\
\hline
\end{tabular}

